\title{
Tuberculosis elimination: theory and practice in Europe
}

\author{
Lia D’Ambrosio ${ }^{1,11}$, Masoud Dara ${ }^{2,11}$, Marina Tadolini ${ }^{3}$, Rosella Centis ${ }^{1}$, \\ Giovanni Sotgiu ${ }^{4}$, Marieke J. van der Werf ${ }^{5}$, Mina Gaga ${ }^{6}$, Daniela Cirillo \\ Antonio Spanevello ${ }^{1,8}$, Mario Raviglione9, Francesco Blasi ${ }^{10}$ and \\ Giovanni Battista Migliori ${ }^{1}$, on behalf of the European national \\ programme representatives
}

Affiliations: 'WHO Collaborating Centre for Tuberculosis and Lung Diseases, Fondazione Salvatore Maugeri, Care and Research Institute, Tradate, ${ }^{3}$ Dept of Medical and Surgical Sciences, Alma Mater Studiorum University of Bologna, Bologna, ${ }^{4}$ Epidemiology and Medical Statistics Unit, Dept of Biomedical Sciences, University of Sassari - Research, Medical Education and Professional Development Unit, AOU Sassari, ${ }^{7}$ Emerging Bacterial Pathogens Unit, San Raffaele Scientific Institute, Milan, ${ }^{8}$ Università degli Studi dell'Insubria, Varese, and ${ }^{10}$ Dipartimento Fisiopatologia Medico-Chirurgica e dei Trapianti, University of Milan, IRCCS Fondazione Cà Granda, Milan, Italy. ${ }^{2}$ World Health Organization, Regional Office for Europe, Copenhagen, Denmark. ${ }^{5}$ European Centre for Disease Prevention and Control, Stockholm, Sweden. ${ }^{67 t h}$ Respiratory Medicine Dept, Athens Chest Hospital, Athens, Greece. ${ }^{9}$ Global TB Programme, World Health Organization, Geneva, Switzerland. ${ }^{11} \mathrm{~L}$. D'Ambrosio and M. Dara contributed equally to the study.

Correspondence: G.B. Migliori, WHO Collaborating Centre for Tuberculosis and Lung Diseases, Fondazione Salvatore Maugeri, Care and Research Institute, via Roncaccio 16, 21049, Tradate, Italy.

E-mail: giovannibattista.miglioridfsm.it

ABSTRACT Although Europe identified the pathway to tuberculosis (TB) elimination in 1990, no information on programmes for country preparedness is available.

A questionnaire investigating TB elimination activities was submitted to 38 national TB programme representatives of low TB incidence ( $<20$ cases per 100000 population) European countries/territories of the World Health Organization European region.

Out of 31 providing a complete answer, 17 (54.8\%) reported to have a dedicated national TB programme, $20(64.5 \%)$ a national plan including TB elimination (13 (41.9\%) including targets), $22(71 \%)$ guidelines, $14(45.2 \%)$ a specific budget for TB activities, and 23 (74.2\%) TB reference centres. All countries reported having case-based electronic TB surveillance, 19 (61.3\%) perform regular supervision, $12(38.7 \%)$ have a monitoring and evaluation plan and five (16.1\%) perform modelling. In three countries $(9.7 \%)$, TB health services are free for insured individuals only. In 22 countries/territories (71\%) not all TB drugs were available, while in $12(38.7 \%)$ drug stock-outs have been described. Although high-risk group screening for latent TB infection is performed by the majority of countries, only $6(19.4 \%)$ provided figures on preventive treatment completion rates.

Not all elements identified as essential for country preparedness to achieve TB elimination are available in the countries surveyed.

@ERSpublications

As TB elimination interventions are sub-optimally applied, more training, awareness and political commitment are necessary http://ow.ly/ru6PV

Received: Nov 142013 | Accepted after revision: Nov 292013 | First published online: Jan 32014

Conflict of interest: None declared.

This article has supplementary material available from www.erj.ersjournals.com 


\section{Introduction}

For tuberculosis (TB) control, the detection and cure of infectious TB patients living in the community represented the main pillar of the national TB programmes for decades, as it aims to reduce transmission of Mycobacterium tuberculosis. This approach, implemented through the World Health Organization (WHO)'s "Stop TB Strategy" has proven to be cost-effective $[1,2]$. Proper diagnosis and rapid treatment of infectious TB cases have saved millions of lives, contributed to curbing the epidemic, and remain the essential interventions of TB control [3,4]. However, as existing strategies have not significantly accelerated incidence decline towards elimination (defined as $<1$ sputum smear positive cases per one million inhabitants) [5-7], the WHO began in 2012 to plan the future post-2015 strategy. It is also aiming at eliminating TB in low-incidence settings through better understanding of the epidemic and the design of effective approaches towards people at high risk of disease [8]. In 2008, the Framework Action Plan to Fight Tuberculosis in the European Union was published [9]. The long-term goal of this plan is to control and ultimately eliminate $\mathrm{TB}$ in the European Union (EU). To reach elimination, diagnosis and preventive treatment of latent TB infection (LTBI) to supplement detection and treatment of active TB disease needs to be emphasised in particular [7,9]. Although LTBI preventive treatment is recommended by almost all existing national guidelines, clinicians and public health specialists still hesitate to implement it on a large scale [7].

While Europe conceptualised TB elimination in 1990 [10], the framework for it was only published in 2002 within the outcomes of the Wolfheze meetings [5]. The core interventions proposed in that document were: 1) ensuring early detection of TB patients and their treatment until cure and preventing avoidable deaths from $\mathrm{TB}$; 2) reducing incidence of infection by risk group management and prevention of transmission of infection in institutional settings; and 3) reducing the prevalence of TB infection through outbreak management and provision of preventive therapy for specified groups and individuals.

A recent European Respiratory Society (ERS) document on TB elimination [7] reinforced the importance of the seven core areas of the European Centre for Disease Prevention and Control (ECDC) Framework Action Plan to Fight Tuberculosis in the European Union [9], i.e. commitment, TB awareness and capacity of health systems, surveillance, quality laboratories and care, capacity to manage drug resistant TB and TB/ HIV co-infection, introduction of new tools and operational research and establishment of international collaborations (online supplementary material, annex 1).

The WHO European region is estimated to be responsible for one of every four multidrug-resistant (MDR)TB patients worldwide. More than $97 \%$ of these cases are in high MDR-TB burden countries in Eastern Europe, which includes some countries of the EU, such as Romania and the Baltic republics. In response to this alarming situation, the WHO European region has developed a consolidated action plan to prevent and combat extensively drug-resistant (XDR)- and MDR-TB for 2011-2015 [11], which was endorsed at the WHO Regional Committee in 2011.

In order to assess in-depth by what extent the principles of the seven core areas mentioned above have been implemented in Europe, a survey was carried out by the ERS and WHO in collaboration with the ECDC [12-14].

\section{Methods}

A comprehensive questionnaire with both closed and open-ended questions was designed by ERS and WHO experts with input by ECDC. Specific questions investigating TB elimination activities were grouped into the seven areas included in the ECDC Framework Action Plan to Fight Tuberculosis in the European Union and discussed by Diel et al. [7] (online supplementary material, annex 1), and included interventions mentioned in WHO documents $[5,12]$.

The questionnaire was submitted to national TB programme representatives via the WHO Office for Europe in January 2013 to all EU countries and the non-EU low TB incidence countries of the WHO European region. Based on a Wolfheze consensus statement, all countries with less than 20 notified TB cases per 100000 population are categorised as low TB incidence countries $[5,7,10] .38$ countries met the criteria and received the questionnaire. Countries that initially had not responded by the deadline (April 2013) were contacted by e-mail and phone and reminded, with the support of ERS delegates representing the national societies. Data were summarised by means of descriptive statistics per each of the elimination areas described in annex 1 in the online supplement. We calculated the population covered by TB reference centres, by dividing the national population (using http://data.worldbank.org/country as source) by the number of references centres in the country. To keep the text short, the number of answers not provided for a given question was not systematically reported in the text (see tables for details). 


\section{Results}

By July 30, 2013, 31 out of $38(81.6 \%)$ invited countries and territories had returned the completed questionnaire. They included Albania, Belgium, Croatia, Cyprus, Czech Republic, Denmark, Estonia, Finland, France, Germany, Greece, Hungary, Ireland, Israel, Italy, Latvia, Malta, the Netherlands, Norway, Poland, Portugal, Republic of Macedonia, Romania, Serbia, Slovakia, Slovenia, Spain, Sweden, Switzerland, UNMIK Kosovo (United Nations Security Council Resolution no.1244) and the UK.

\section{1) TB control commitment, TB awareness and capacity of health systems}

Out of the 31 countries and territories surveyed, 17 (54.8\%) reported having a national TB coordinating body or a dedicated national TB programme (CB/NTP), $20(64.5 \%)$ a national plan which includes TB elimination, $22(71 \%)$ have guidelines and 14 (45.2\%) only have a specific budget for TB (table 1 and annex 2 in the online supplement). The Netherlands and Switzerland reported all elements that show country commitment to TB control, i.e. a CB/NTP, a national/subnational plan, guidelines and a specific budget for TB.

Of the 20 countries/territories with a CB/NTP, only 13 (65\%) (Albania, Croatia, Estonia, Hungary, Israel, Latvia, Malta, Republic of Macedonia, Romania, Serbia, Slovenia, UNMIK Kosovo and UK) declared that the plan includes targets for TB control and/or elimination.

$23(74.2 \%)$ countries/territories declared to have TB reference centres. Czech Republic, Estonia, Germany, Greece, Hungary, Israel, Italy, Latvia, Poland, Portugal, Republic of Macedonia, Slovakia, Slovenia, the Netherlands, Switzerland and UNMIK Kosovo reported one to two reference centres; Cyprus, Denmark, Ireland, Malta and Romania reported three to seven; Albania 28 and France 150. TB reference centres covered on average a population of 9.7 million (range 0.1 to 82 million). Eight of 31 (25.8\%) countries did not report having TB reference centres (Belgium, Croatia, Finland, Norway, Serbia, Spain, Sweden and UK).

\section{2) Surveillance}

All countries (except the administrative territory of Serbia-UNMIK Kosovo) reported case-based electronic TB surveillance data collection at national level (table 2 and annex 3 in the online supplement). 10 countries (32.3\%) (Belgium, Czech Republic, Estonia, Finland, France, Israel, Italy, Republic of Macedonia, Serbia and Slovenia) reported use of a combination of paper forms, electronic database and electronic internetbased database; eight countries (25.8\%) (Greece, Hungary, Ireland, Latvia, the Netherlands, Portugal, Romania and Sweden) reported having an electronic internet database; six countries (19.4\%) (Albania, Denmark, Norway, Poland, Slovakia and Switzerland) had paper forms and an electronic database; three countries/territories (9.7\%) (Croatia, Cyprus and UNMIK Kosovo) had paper forms only; two countries (6.5\%) (Spain and Malta) had an electronic database; the UK had paper forms and an electronic internet database, while Germany had an electronic database and electronic internet-based database.

Out of 31 countries/territories, 18 (58.1\%) have dedicated TB surveillance staff. In all countries, except Cyprus, data validation and quality control activities are implemented. Supportive supervision from, for example, the national level to a sub-national level is performed in 19 (61.3\%) countries. All countries/ territories reported that their surveillance data are regularly analysed and that the results are reported.

Only 12 out of 31 countries/territories (38.7\%) (Albania, Finland, Germany, Greece, Hungary, Israel, Republic of Macedonia, Malta, Romania, Serbia, Slovakia and UNMIK Kosovo) reported having monitoring and evaluation plans. Five countries only (16.1\%) (Cyprus, Germany, Hungary, Slovakia and Slovenia) have developed mathematical modelling to predict future epidemiological trends, while Norway conducted it in 1996 for the last time.

\section{3) Laboratory services}

All countries/territories except Malta have TB reference laboratories, and all but France, Greece, Malta, Poland and Switzerland are supervised by a supra-national reference laboratory that is part of the WHO network (table 3 and annex 4 in the online supplement). An external quality assurance system is in place in all countries except Malta.

Microscopy, culture, and drug susceptibility testing (DST) is performed in 20 countries (64.5\%). The number of laboratories that perform microscopy ranges from 0.1 to 7.9 per million population. While the number of laboratories performing culture ranges from 0.5 to 10.2 per million population, for laboratories performing DST the range is 0.1 to 1.9 per million population. Xpert MTB/RIF is reported as not being available on a national level in Albania, Denmark, Malta, Republic of Macedonia, Romania, Serbia, Spain and Sweden (25.8\%), whereas WHO-endorsed rapid molecular diagnostics other than Xpert MTB/RIF are available in 24 of the $30(77.5 \%)$ countries/territories that provided information. 

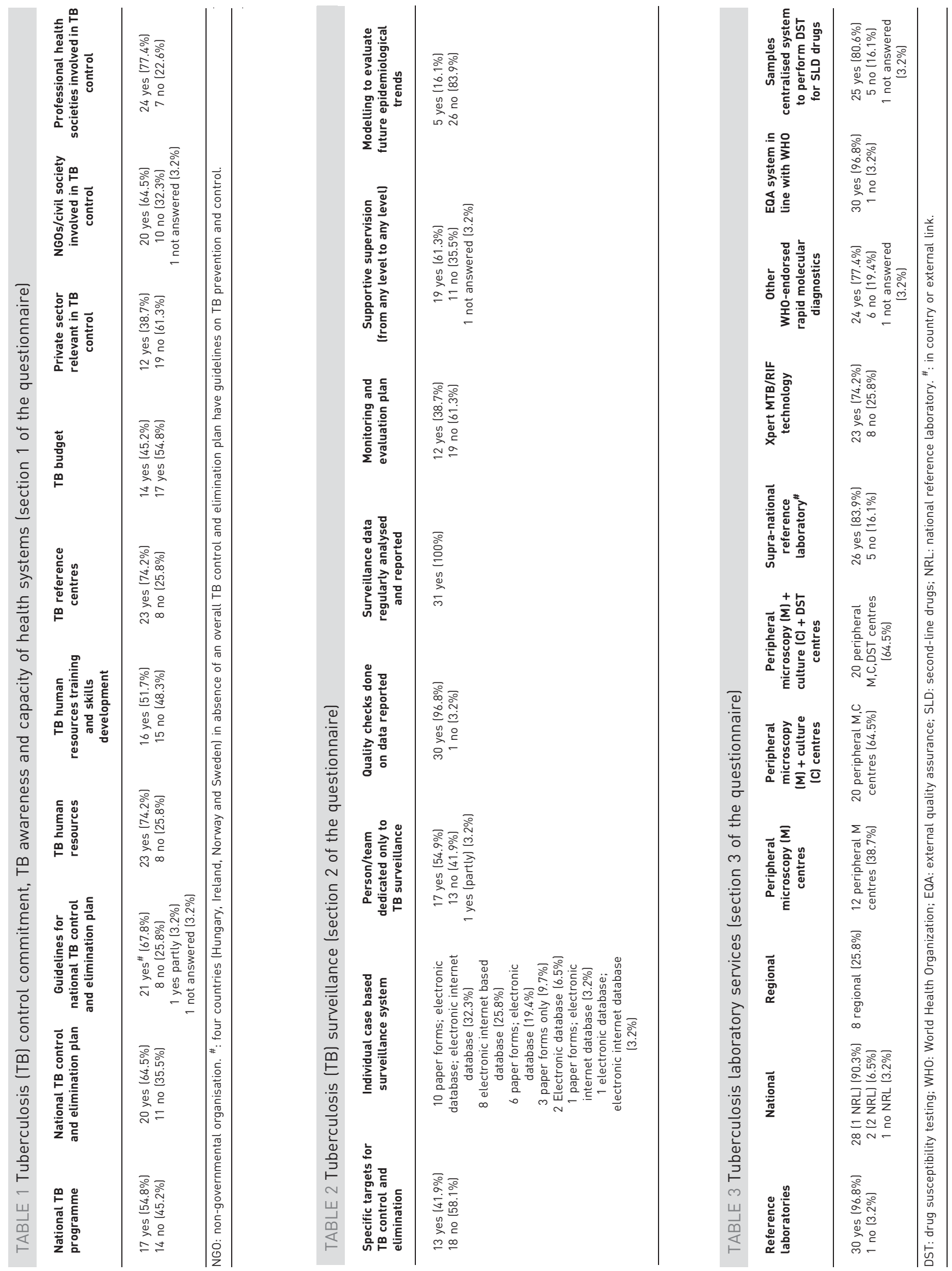


\section{4) Prompt and high-quality TB care for all}

Free healthcare for all TB cases (diagnosis and treatment) is the standard in 27 countries/territories (87.1\%), while in Czech Republic, the Netherlands and Switzerland, health services are free for insured individuals only and in Cyprus out-patient services need a nominal fee to be paid (table 4 and annex 5 in the online supplement). 10 countries/territories (32.2\%) (Estonia, Israel, Latvia, Malta, the Netherlands, Republic of Macedonia, Serbia, Switzerland, UNMIK Kosovo and the UK) provide incentives and enablers to TB patients. All of these countries/territories but Estonia, Israel and Latvia, plus Germany, Hungary, Ireland, Norway and Spain use treatment supporters to assist TB patients with taking their TB treatment.

Access to first- and second-line anti-TB drugs is not equal in all countries responding to the questionnaire. In 22 countries/territories (71\%) not all TB drugs were available. 12 countries (38.7\%) (Czech Republic, Denmark, Estonia, Greece, Malta, the Netherlands, Poland, Portugal, Slovakia, Slovenia, Sweden and the UK) reported to had stock-outs of at least some of the first- and/or second-line drugs in the past 2 years.

The organisation of TB prevention and control is reported to be the same in the prison system and the civilian sector in $28(90.3 \%)$ countries (table 4). In France and UNMIK Kosovo the civilian and prison system for TB prevention and control is different.

All countries/territories except Croatia, Cyprus, Greece and Latvia have mentioned to have specific strategies to manage risk groups.

\section{5) M/XDR TB and TB/HIV co-infection}

Out of 31 countries/territories, 13 (41.9\%) manage MDR-TB cases mainly in hospitals. 17 (54.9\%) countries/territories use a combined approach, in which they manage MDR-TB cases partly as in-patients and partly as out-patients (i.e. a 6-9 months stay in hospital when patient is infectious, followed by an outpatient phase) (table 5 and annex 6 in the online supplement). Reference centres or hospital departments for MDR-TB were available in 24 countries/territories (77.4\%).

Absent or very limited collaboration between TB and HIV caregivers at a programme and clinical level was reported by a third of countries/territories (35.5\%): Belgium, Czech Republic, Greece, Italy, the Netherlands, Norway, Poland, Spain, Switzerland, UNMIK Kosovo and the UK. This is also reflected by the fact that only 12 countries (38.7\%) reported to have one stop services for the diagnosis and treatment of TB and HIV.

\section{6) New tools and operational research}

A strategy for the introduction and implementation of new tools for TB control was available in eight countries (25.8\%): Estonia, France, Germany, Hungary, Latvia, Republic of Macedonia, Slovakia and the UK.

Only four (12.9\%) countries/territories (Hungary, Latvia, the Netherlands and UNMIK Kosovo) reported having an operational research plan, while 14 (45.2\%) actually performed operational research on TB. Three countries $(9.7 \%)$ had a specific budget allocated to operational research.

In terms of LTBI management (table 6 and annex 7 in the online supplement), 22 countries (71\%), except Albania, Croatia, Denmark, Germany, Greece, Romania, Serbia, Slovenia and UNMIK Kosovo, reported to perform screening for LTBI in high risk groups (contacts, HIV positive and other immunosuppressed individuals, patients prior to anti-tumour necrosis factor- $\alpha$ administration, migrants, asylum seekers, prisoners, children and adolescents, health care workers, drug addicts, etc.).

All countries/territories performed contact tracing and also reported to treat LTBI (except Norway, which has no official data, Czech Republic where only children are treated, and UNMIK Kosovo which did not answer). Six (19.4\%) countries (Greece, the Netherlands, Portugal, Republic of Macedonia, Slovakia and Switzerland) were able to provide figures on LTBI preventive treatment completion rates, which ranged between 40 and $88 \%$.

An infection control plan was available in 21 out of 31 countries/territories (67.8\%), while seven countries (22.5\%) (i.e. Belgium, France, Italy, Poland, Serbia, Spain and Switzerland) have either drafts or national guidelines or recommendations or control measures for TB infection control included in the national surveillance guidelines. Three countries (9.7\%) reported to have no plans (Greece, Portugal and Republic of Macedonia). Infection control measures were implemented in 26 countries/territories (83.9\%).

\section{7) Partnership and collaboration with countries}

Few countries (Belgium, Estonia, Germany, Latvia, the Netherlands, Serbia and Spain) declared to have international strategic collaborations with other countries that support TB control (table 7 and annex 8 in the online supplement). 

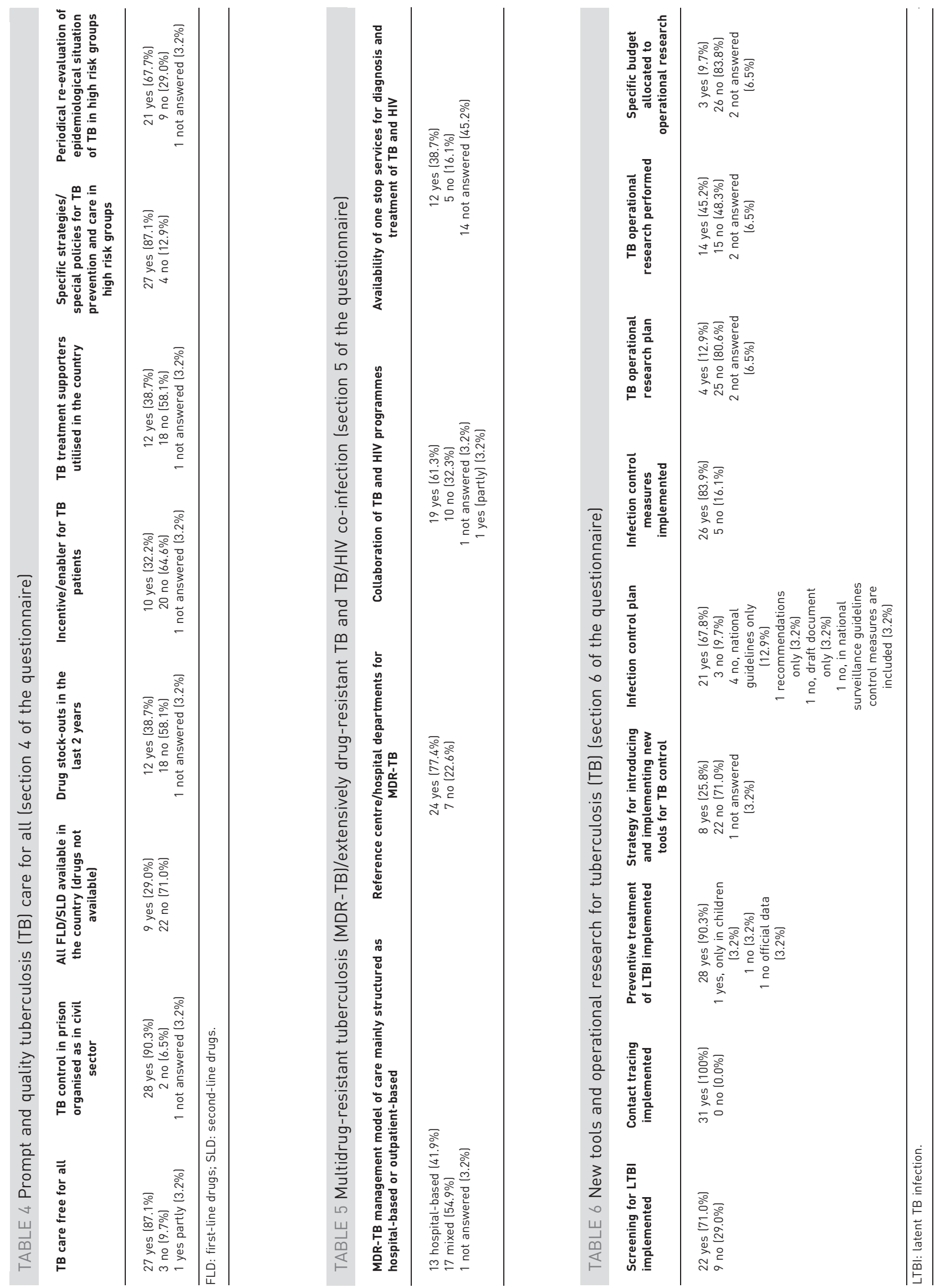
TABLE 7 Partnership and consilium for tuberculosis (TB) (section 7 of the questionnaire) available in $20(64.5 \%)$ countries/territories.

\section{Discussion}

Delegates for European countries recommended the pathway towards TB elimination in 1990, during the first Wolfheze Conference, convening experts from the European TB low incidence countries, and USA, Canada and Japan [10]. We present the results of the ERS/WHO/ECDC survey on TB elimination, which aimed to measure by what extent the recommended interventions and systems needed to reach $\mathrm{TB}$ elimination are implemented in low TB incidence countries of the WHO European region.

This study represents the first international survey investigating in a systematic manner how core interventions necessary to reach elimination are being implemented.

The results of this survey show that European countries need to work more intensively, prepare consolidated plans and ensure available resources to move towards elimination. While most countries are well covered for the majority of the elimination interventions the survey also showed the necessity to embark further on interventions which are not yet fully implemented in Europe, including diagnosis and preventive treatment of LTBI, and an evaluation system of preventive therapy adherence/compliance rates $[5,7]$. In fact, while $70 \%$ of the European countries screens certain at-risk persons or populations for LTBI (although with different methods and targets $[15,16]$ ), less than $20 \%$ of them collects information on LTBI preventive treatment outcomes. This information is often collected at the peripheral level and on individuals accepting LTBI preventive treatment only. In very few countries is it possible to analyse LTBI data at national level and know the proportion of those who declined preventive treatment. A national register of all latently infected individuals diagnosed, initiating preventive treatment and including data on preventive treatment completion is a necessary monitoring and evaluation tool, allowing estimation of the public health impact of LTBI preventive treatment and impact evaluation of the intervention, on top of its individual clinical benefit.

A systematic assessment of results potentially achievable through the different interventions available today, including adoption of new tools in diagnosis (rapid molecular testing for TB and drug resistance) and treatment (introduction of new drugs effective against MDR-TB), adoption on a wide scale of LTBI diagnosis and preventive treatment, and improved access to care for high-risk populations (including free diagnosis and treatment, as well as social protection mechanisms preventing income loss) is the first step towards building an elimination plan together with a proper assessment of the status of the epidemic and the populations most affected. Assumptions around the potency of new tools in the pipeline that may become available in the next decade need also to be factored in during a projection exercise targeting elimination. Mapping of the burden in each country looking at epidemiological, social and economic factors will allow proper planning of interventions in different areas and settings. Eventually, projections through quantitative analysis on these assessments and consequent modelling of various scenarios will reveal what is achievable under different assumptions of implementation capacity and new tool developments over the next few decades, thus allowing monitoring of step by step progress towards established elimination targets $[5,7,10,17]$.

Although most European countries/territories declare having a national TB programme, a TB control and elimination plan and guidelines on how to implement it, not all of them have formally included elimination into their national policies, and 18 out of $31(58.1 \%)$ have no specific targets for TB control and elimination.

Less than half of the countries have a TB-specific national budget. Although a TB-specific national budget is not essential in view of a Europe decentralised primary care system, it is vital that the national plan and strategy for TB prevention and control contain a budget including sources of funding for the identified activities, since funding is considered a positive reflection of government commitment [2, 18]. The mechanisms to fund TB control and elimination activities varies across the target countries, due to low prevalence of the disease and integration of diagnosis and treatment services in general healthcare, and the different priority TB has in the political agenda. As a result, several activities including human resources 
development, involvement of the private sector and civil society in TB control and elimination are implemented in an ad hoc manner in the target countries of this survey.

Similarly country-specific is the organisation of the different European programmes in terms of diagnostic and treatment reference centres, and their catchment populations.

The vast majority of the countries in Europe has developed sound electronic, case-based surveillance systems managed by specialised staff, all of them performing quality checks (except Cyprus) and all regularly analysing and reporting surveillance data to WHO and ECDC. Interestingly, on the negative side, only $12(38.7 \%)$ countries/territories have a monitoring and evaluation plan and 19 (61.3\%) perform supervision at the different levels of the health system.

Almost all countries reported to have an external quality assurance system, implemented through the WHO network of supranational reference laboratories and other networks. Almost all countries/territories (25 out of $31,80.6 \%$ ) have been able to implement a centralised system performing DST for all first- and secondline TB drugs and determining the prevalence of drug resistance at the national level. 23 (74.2\%) countries/ territories out of 31 have implemented the Xpert MTB/RIF technology, as recommended by the EU standards for TB care and by WHO [3, 8, 19].

In three out of 31 countries/territories $(9.7 \%) \mathrm{TB}$ care is not yet free for all patients. The public health importance of ensuring affordable access to TB care of all individuals with $\mathrm{TB}$ disease, and prevention for those with LTBI, has been underlined in several international documents $[5,20]$. The fact that TB care is not universally free may seriously undermine TB elimination. It should be assessed whether affordable access to TB prevention and care that avoids catastrophic expenditure for the person or family affected is guaranteed in countries where TB care is not free of charge for all. In addition, a supportive health system would have provision for the care of diseases relevant for public health among illegal immigrants and other at-risk populations for whom there is no incentive to seek care.

In 22 countries/territories (71\%) not all TB drugs were available, while 12 countries $(38.7 \%)$ admitted the presence of drug stock-outs in the past 2 years. This finding, in a way surprising in Europe, has been described already in a previous ECDC survey [21].

Availability of drugs relies on several factors in addition to financial resources, including the legal framework, registration procedures and interest of pharmaceutical companies to commercialise the product in a given country. A European or sub-regional plan to make all anti-TB drugs available at least to treat MDR-/XDR-TB cases has been discussed but is difficult to implement [21].

Achieving high level treatment outcomes of both drug-susceptible and drug-resistant cases is an important pre-requisite for achieving TB elimination. 20 countries $(64.6 \%)$ do not report promoting adherence by using incentives or enablers, while $18(58.1 \%)$ do not propose treatment supporters to enhance treatment outcomes. The result of this survey also alludes to previous findings and surveillance data which show poor treatment outcome of MDR-/XDR-TB in many settings [22]. Best practices in promoting treatment adherence need to be further investigated, discussed and promoted at the European level, as treatment success rates in the WHO European region are still low when compared with the other regions [23].

Successful management of TB in marginalised populations is extremely important.

28 out of $31(90.3 \%)$ countries/territories have the same approach to TB control in the prison and in the civilian sector, and have specific policies to deal with risk groups (although in nine countries risk groups are not periodically re-evaluated based on the epidemiological evidence).

Different models of MDR-TB and TB/HIV care are available, with half of the countries/territories having a clinical management system based on both in- and out-patient care. A patient-centred approach ensuring, to the extent possible, home care will potentially reduce suffering/difficulties and costs for patients and their families, enhance treatment adherence and reduce Mycobacterium tuberculosis transmission in institutional settings $[3,24]$.

Seven (22.6\%) countries have no MDR-TB reference centre, while 10 countries/territories (32.3\%) reported not to have any collaboration between TB and HIV/AIDS programmes, five (16.1\%) not having a one stop service for diagnosis and treatment of TB and HIV co-infected individuals.

The capacity to limit transmission of $M$. tuberculosis from infectious individuals and to introduce new diagnostic and treatment tools in a rational manner at scale are of paramount importance from an elimination perspective. The majority of countries/territories have an infection control plan (21 out of 31, $67.8 \%$ ), but only four countries/territories have an operational research plan (three of them with specific funds) and eight developed a plan to introduce and implement new tools for TB control. 
Despite the finalisation of the Consensus Paper on Minimum Package of Cross Border TB Control and Care [20], only seven countries have plans to collaborate with other countries in different ways (technical assistance, programme reviews, international collaboration between laboratories or MDR-TB reference centres, joint training activities, etc.). With increasing movement of populations, including migration and business or leisure travel, enhanced cross-border collaboration and cooperation with early case-finding and treatment follow-up is necessary. Last but not least, the majority of countries/territories declared having a clinical consultation system in place (TB consilium) to ensure the correct management of difficult-to-treat TB cases [12-14, 25]. The correct management of drug-resistant and other complicated cases will, in fact, prevent the development of further drug resistance.

To obtain a correct overview of the TB prevention and control situation in European countries with $<20 \mathrm{~TB}$ cases per 100000 population, the questionnaire survey was carefully designed, pre-tested and administered through WHO national representatives. To limit inaccuracies in the responses all unclear answers, unanswered questions or doubts expressed by the respondents were rapidly discussed with the respondent by the coordinating team by e-mail or telephone. From discussion with the respondents we learned that non-response to certain questions can be due to multiple reasons, including the national correspondents not being able to generalise the response due to different situations in their diverse settings or administrative divisions, or by the absence of official figures. Furthermore we cannot exclude a potential bias in the answers related to country-specific differences in interpreting the questions posed in the questionnaire.

However, the results of this study are the first and (as of today) the only available snapshot on TB elimination practices in low TB incidence countries in Europe, and provide important information for further clinical and public health action to improve TB control and, hopefully, reach the elimination phase (i.e. $<1$ sputum smear positive cases per one million inhabitants) in Europe.

On top of the three prevention activities (improve diagnosis of LTBI; improve regimens to treat LTBI; ensure public health commitment to make them possible) $M$. tuberculosis transmission needs to be cut via early case detection and screening in order to achieve elimination.

It remains to be seen whether the currently available tools, diagnostics, drugs and vaccines, are sufficient to meet TB elimination targets in Europe. We will certainly need better tools to identify those individuals with TB infection that have a high risk of developing TB disease. Although research is ongoing to identify new biomarkers, thus far no candidates have proven to be of added value [7]. Also, preventive treatment of LTBI is lengthy and has adverse effects, both impeding completion of LTBI preventive treatment. New drugs or drug combinations (e.g. bedaquiline, PA-824 and delamanid [26-30]) and existing drugs with new indications (e.g. rifapentine [31]) may be needed to improve LTBI preventive treatment completion rates [32-34].

A cost-effective and rational introduction of new diagnostics and drugs in national programmes is needed, so that TB disease and infection are correctly diagnosed and new drugs preserve their efficacy [35].

Finally, a vaccine that prevents TB infection, or the development of TB disease after infection among already infected individuals, would be fundamental in the efforts to reach TB elimination. Like other efficacious vaccines, it should be safe, inexpensive, easy to administer (schedule, route, etc.), targeted to the entire population, and able to trigger a cytotoxic response as well as a long-term memory T-cell repertoire.

Both adequate therapeutic management and TB immunisation with a new vaccine will hinder the emergence and spread of drug-resistant strains. However, such a vaccine may not be available for another decade or more $[36,37]$.

Sound and bold policies need to be implemented at the national level in Europe to ensure that individuals with active TB are detected promptly and treated effectively to rapidly eliminate transmission to others, and that people with LTBI at high risk of active TB are identified and offered preventive treatment. Ultimately, to reach elimination, the whole package of necessary core interventions must be duly put into practice [38]. Without preparedness, proper planning and resources, elimination of TB in Europe remains as elusive as it was in 1990.

\section{Acknowledgements}

The national representatives who contributed to collect the survey were: Hasan Hafizi (Albania); Maryse Wanlin, Wouter Arrazolade Oñate, Guido Groenen (Belgium); Vera Katalinić Janković, Aleksandar Šimunović (Croatia); Jiri Wallenfels (Czech Republic); Constantia Voniatis (Cyprus); Peter Henrik Andersen (Denmark); Piret Viiklepp, Manfred Danilovits, Tiina Kummik (Estonia); Petri Ruutu (Finland); Thierry M. Comolet (France); Walter Haas, Gerit Korr, Sabine RüschGerdes, Torsten Bauer, Barbara Hauer, Bonita Brodhun (Germany); Mina Gaga, Konstantinou Papavenstsis, Spala Papadima (Greece); Zsofia Pusztai (Hungary); Joan O’Donnell (Ireland); Daniel Chemtob (Israel); Enrico Girardi (Italy); Rukije Mehmeti (UNMIK Kosovo); Vija Riekstina (Latvia); Analita Pace Asciak (Malta); Trude M. Arnesen (Norway); Ewa Augustynowicz-Kopeć, Maria Korzeniewska-Kosela, Paulina Marianna Miskiewicz, Dominika Miecznikowska (Poland); Raquel Duarte, Ana Maria Correia, Antonio Manuel S.C. Diniz (Portugal); Stefan Talevski, Maja Zakoska 
(Republic of Macedonia); Gilda Popescu, Domnica Chiotan (Romania); Gordana Radosavl Jevic Asic (Serbia); Ivan Solovic (Slovakia); Marijan Ivanuša, Mitja Košnik (Slovenia); Elena Rodríguez Valín, Odorina Tello Anchuela (Spain); Jerker Jonsson (Sweden); Peter Helbling, Jean Pierre Zellweger (Switzerland); Gerard de Vries, Connie Erkens (the Netherlands); Laura Anderson, Ian Laurenson (UK).

\section{References}

Sotgiu G, Spanevello A, Migliori GB. History of tuberculosis and drug resistance. NEJM 2013; 368: 88-89.

Raviglione M, Marais B, Floyd K, et al. Scaling up interventions to achieve global tuberculosis control: progress and new developments. Lancet 2012; 379: 1902-1913.

3 Migliori GB, Zellweger JP, Abubakar I, et al. European Union standards for tuberculosis care. Eur Respir J 2012; 39: 807-819.

4 Hopewell PC, Pai M, Maher D, et al. International standards for tuberculosis care. Lancet Infect Dis 2006; 6: 710-725.

5 Broekmans JF, Migliori GB, Rieder HL, et al. European framework for tuberculosis control and elimination in countries with a low incidence. Recommendations of the World Health Organization (WHO), International Union Against Tuberculosis and Lung Disease (IUATLD) and Royal Netherlands Tuberculosis Association (KNCV) Working Group. Eur Respir J 2002; 19: 765-775.

6 Veen J, Migliori GB, Raviglione M, et al. Harmonisation of TB control in the WHO European region: the history of the Wolfheze Workshops. Eur Respir J 2011; 37: 950-959.

7 Diel R, Loddenkemper R, Zellweger J-P, et al. Old ideas to innovate TB control: preventive treatment to achieve elimination. Eur Respir J 2013; 42: 785-801.

8 World Health Organization. Report of the 13th Meeting WHO Strategic and Technical Advisory Group for Tuberculosis (STAG-TB) 11-12 June 2013. Document WHO/HTM/TB/2013.09. Geneva, World Health Organization, 2013.

9 European Centre for Disease Prevention and Control. Framework Action Plan to Fight Tuberculosis in the European Union. Stockholm, ECDC, 2008.

10 Clancy L, Rieder HL, Enarson DA, et al. Tuberculosis elimination in the countries of Europe and other industrialized countries. Eur Respir J 1991; 4: 1288-1295.

11 WHO Regional Office for Europe. Roadmap to Prevent and Combat Drug-resistant Tuberculosis. The Consolidated Action Plan to Prevent and Combat Multidrug- and Extensively Drug-resistant Tuberculosis in the WHO European Region, 2011-2015. Copenhagen, World Health Organization, Regional Office for Europe, 2011.

12 Blasi F, Barnes PJ, Gaga M, et al. Future Directions for the ERS: Presidential plans. Eur Respir J 2013; 42: 875-880.

13 Blasi F, Reichman LB, Migliori GB. Presenting the European Forum for TB Innovation: innovative thinking in progressing towards TB elimination in Europe. Eur Respir J 2012; 40: 806-808.

14 Blasi F, Dara M, van der Werf MJ, et al. Supporting TB clinicians managing difficult cases: the ERS/WHO Consilium. Eur Respir J 2013; 41: 491-494.

15 Sotgiu G, Bourdin Trunz B, Migliori GB, et al. Childhood tuberculosis outbreaks in EU/EEA: a systematic review. Eur Respir J 2013; 42: Suppl. 57, 336s.

16 Migliori GB, Centis R, D'Ambrosio L, et al. Impact and management of TB childhood outbreaks in EU/EEA. Eur Respir J 2013; 42: Suppl. 57, 724s.

17 Dye C, Raviglione MC. Weigh all TB risks. Nature 2013; 502: S13.

18 Raviglione MC, Uplekar MW. WHO's new Stop TB Strategy. Lancet 2006; 367: 952-955.

19 Migliori GB, Sotgiu G, Blasi F, et al. Towards the development of EU/EEA Standards for Tuberculosis Care (ESTC). Eur Respir J 2011; 38: 493-495.

20 Dara M, de Colombani P, Petrova-Benedict R, et al. Minimum package for cross-border TB control and care in the WHO European region: a Wolfheze consensus statement. Eur Respir J 2012; 40: 1081-1090.

21 Sotgiu G, D’Ambrosio L, Centis R, et al. Availability of anti-tuberculosis drugs in Europe. Eur Respir J 2012; 40: 500-503.

22 European Centre for Disease Prevention and Control/WHO Regional Office for Europe. Tuberculosis Surveillance and Monitoring in Europe 2012. Stockholm, European Centre for Disease Prevention and Control, 2012.

23 World Health Organization. Global Tuberculosis Report 2012. Document WHO/HTM/TB/2012.6. Geneva, World Health Organization, 2012.

24 Sotgiu G, D'Ambrosio L, Centis R, et al. TB and M/XDR-TB infection control in European TB reference centres: the Achilles' heel? Eur Respir J 2011; 38: 1221-1223.

25 D'Ambrosio L, Tadolini M, Dupasquier S, et al. ERS/WHO Tuberculosis Consilium: reporting on the initial 10 cases. ERJ 2014; 43: 286-289.

26 Gler MT, Skripconoka V, Sanchez-Garavito E, et al. Delamanid for multidrug-resistant pulmonary tuberculosis. N Engl J Med 2012; 366: 2151-2160.

27 Skripconoka V, Danilovits M, Pehme L, et al. Delamanid improves outcomes and reduces mortality for multidrugresistant tuberculosis. Eur Respir J 2013; 41: 1393-1400.

28 Diacon AH, Dawson R, von Groote-Bidlingmaier F, et al. 14-day bactericidal activity of PA-824, bedaquiline, pyrazinamide, and moxifloxacin combinations: a randomised trial. Lancet 2012; 380: 986-993.

29 Horsburgh CR Jr, Haxaire-Theeuwes M, Lienhardt C, et al. Compassionate use of and expanded access to new drugs for drug-resistant tuberculosis. Int J Tuberc Lung Dis 2013; 17: 146-152.

30 Tiberi S, De Lorenzo S, Centis R, et al. Bedaquiline in MDR-/XDR-TB cases: first experience on compassionate use. Eur Respir J 2014; 43: 289-292.

31 Sterling TR, Villarino ME, Borisov AS, et al. Three months of rifapentine and isoniazid for latent tuberculosis infection. N Engl J Med 2011; 365: 2155-2166.

32 Sotgiu G, Centis R, D’Ambrosio L, et al. Efficacy, safety and tolerability of linezolid containing regimens in treating MDR-TB and XDR-TB: systematic review and meta-analysis. Eur Respir J 2012; 40: 1430-1442.

33 De Lorenzo S, Alffenaar JW, Sotgiu G, et al. Efficacy and safety of meropenem/clavunate added to linezolid containing regimens in the treatment of M/XDR-TB. Eur Respir J 2013; 41: 1386-1392.

34 Alsaad N, van Altena R, Pranger AD, et al. Evaluation of co-trimoxazole in treatment of multidrug-resistant tuberculosis. Eur Respir J 2013; 42: 504-512. 
Zumla A, Blasi F, Raviglione M. Rational use of anti-tuberculosis drugs in the EU: better patient care and less drug resistance. Eur Respir J 2011; 39: 802-804.

36 Abu-Raddad LJ, Sabatelli L, Achterberg JT, et al. Epidemiological benefits of more-effective tuberculosis vaccines, drugs, and diagnostics. Proc Natl Acad Sci USA 2009; 106: 13980-13985.

37 Dye C, Williams BG. Eliminating human tuberculosis in the twenty-first century. J R Soc Interface 2008; 23: 653-662.

38 Borgdorff MW, van den Hof S, Kremer K, et al. Progress towards tuberculosis elimination: secular trend, immigration and transmission. Eur Respir J 2010; 36: 339-347. 